\title{
GERAÇÃO DE RESÍDUOS SÓLIDOS URBANOS NO SEMIÁRIDO BRASILEIRO: ANÁLISE DE SUSTENTABILIDADE A PARTIR DA APLICAÇÃO DA PEGADA ECOLÓGICA
}

\author{
Paulo Henrique de Almeida Cartaxo ${ }^{1}$, Igor Revelles Gomes Luna ${ }^{1}$, Izaias Romário Soares do Nascimento ${ }^{1}$, Maciel Rocha da Silva ${ }^{1}$, \\ Kardelan Arteiro da Silva ${ }^{2}$, João Paulo de Oliveira Santos ${ }^{*}$ \\ ${ }^{1}$ Universidade Federal da Paraíba, 58397-000, Areia, Brasil. \\ 2 Universidade Federal Rural de Pernambuco, 52171-900, Recife, Brasil.
}

*E-mail:jpos@agro.adm.br

\section{RESUMO}

A grande urbanização e o crescimento significativo da população mundial têm levado a um aumento considerável na geração de resíduos sólidos urbanos (RSU). A destinação final desses materiais é motivo de preocupação por parte da comunidade internacional, principalmente por seu potencial de degradação ambiental. Nesse sentido, visto a carência de estudos quanto à geração de RSU e aplicação de indicadores de sustentabilidade na geração desses materiais em pequenos municípios brasileiros, o presente trabalho objetivou avaliar a aplicação da Pegada Ecológica (PE) para os RSU gerados no município de Poço das Trincheiras, Alagoas. Os dados relativos à geração e gravimetria foram obtidos através do Plano Estadual de Resíduos Sólidos do Estado de Alagoas. Foram quantificados a PE per capita e total, bem como a biocapacidade e saldo ecológico da área de estudo. Observou-se uma taxa de geração de RSU per capita em Poço das Trincheiras da ordem de 0,57 quilogramas/dia, totalizando uma produção anual de 496,4 toneladas, com predominância de material orgânico. A PE per capita dos RSU ficou em 0,1159 gha, já a PE total foi de 276,84 gha. A biocapacidade local foi estimada em 26208 gha, permitindo a obtenção de um superávit ecológico de 25931,16 gha. A Pegada Ecológica mostrou-se uma ferramenta importante para se compreender o grau de impacto da geração de RSU.

Palavras-chave: Qualidade Ambiental. Biocapacidade. Saldo Ecológico.

\section{Introdução}

O planeta Terra passou por uma série de transformações desde a revolução industrial, principalmente no que tange ao grande desenvolvimento econômico e expressivo crescimento populacional [1]. Esses fatores, entre tantas outras alterações sobre o meio, levaram a um aumento da geração de resíduos sólidos urbanos (RSU) em todo o mundo. Um dos grandes gargalos no manejo dos RSU se dá pela sua destinação final, que além de levar a degradação do solo e da água, contribui para muitos outros problemas ambientais, como o aquecimento global [2].

Dessa forma, a geração acentuada de RSU leva concomitantemente a um aumento nas emissões de gases de efeito estufa (GEE) associado ao gerenciamento desses materiais. No Brasil, as taxas de emissões provenientes de RSU registraram aumento entre 2000 e 2015, representando aproximadamente 3,5\% das emissões nacionais de GEE durante esse período [3].

Nesse sentido, percebe-se um aumento da preocupação sobre as contribuições antrópicas ao aquecimento global gerada a partir da gestão de resíduos sólidos, o que tem estimulado a realização de estudos e esforços visando quantificar e reduzir as emissões desse setor [4]. Assim, buscando-se contabilizar o grau de uso do capital natural e sua consequente degradação, diversos instrumentos foram desenvolvidos, com destaque para os indicadores de sustentabilidade. Esses indicadores são ferramentas úteis para auxiliar tomadas de decisão na área de gestão ambiental, podendo ser aplicados para monitorar os processos que envolvam a geração e a deposição de RSU [5].

Um dos indicadores mais usados na atualidade e para diversas finalidade é a Pegada Ecológica (PE), estabelecida por Wackernagel e Rees na década de 1990. A PE permite o estabelecimento de uma nova perspectiva na avaliação do desenvolvimento sustentável, motivo pelo qual tem sido amplamente aplicada por estudiosos da área ambiental [6]. Esse indicador representa a quantidade de área necessária para prover o total de recursos consumidos por uma determinada população, bem como, a área total de terra biologicamente produtiva necessária para absorver os resíduos gerados por esses mesmos indivíduos [7]. Assim, a PE reflete de forma direta a capacidade de carga dos sistemas ambientais, trazendo à tona as características espaciais da apropriação de um ecossistema e o estado das interações entre esse ecossistema e o homem [8]. 
Nesse contexto, tomando como base a carência de estudos quanto à geração de RSU em pequenas cidades do Brasil e a utilização de indicadores de sustentabilidade na geração destes, o presente trabalho objetivou avaliar a aplicação da Pegada Ecológica para os RSU gerados no município de Poço das Trincheiras, Semiárido de Alagoas. Buscando-se assim, determinar o grau de sustentabilidade para a produção desses materiais no supracitado município.

\section{Material e métodos}

O município de Poço das Trincheiras está localizado no sertão do estado de Alagoas (Figura 1), distando aproximadamente 221 km da capital Maceió. Ocupa uma área de $284,25 \mathrm{~km}^{2}$ e possui uma população estimada de 14.354 habitantes [9], dos quais 2388 moram na zona urbana [10].

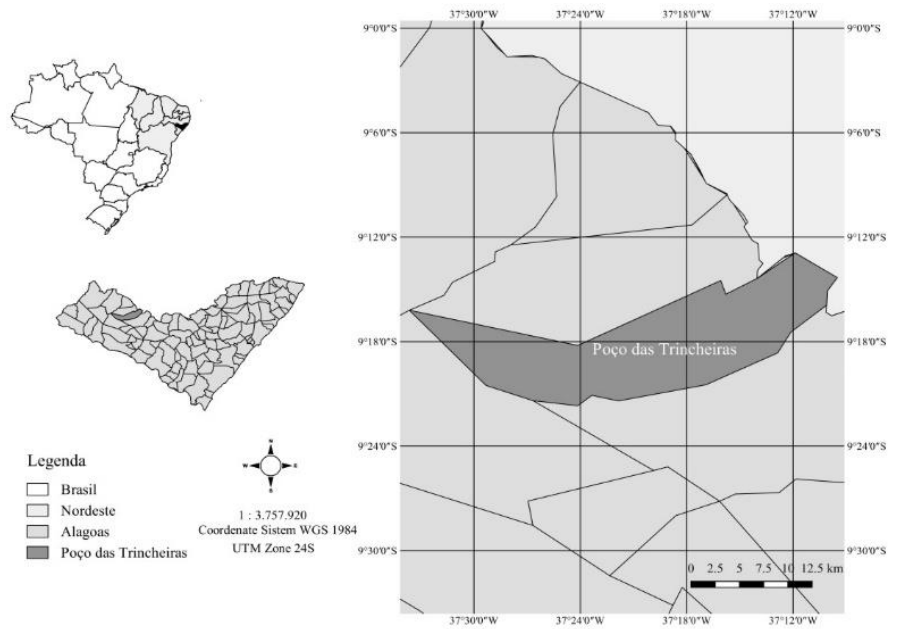

Figura 1 - Localização do município de Poço das Trincheiras, Alagoas.

Os dados de geração de resíduos sólidos urbanos no município de estudo e sua respectiva gravimetria foram obtidos através do Plano Estadual de Resíduos Sólidos do Estado de Alagoas [10].

Para a determinação da PE usualmente se consideram seis tipos de uso da terra: terra cultivável, pastagem, terra construída, mar, áreas florestais e áreas de absorção de carbono. Dessa forma, o consumo de recursos, produção e serviços é convertido em terras bioprodutivas, utilizando para isso fatores de equivalência [8].

A Pegada Ecológica da geração de RSU foi calculada levando em consideração a área necessária para absorver o $\mathrm{CO}_{2}$ emitido na degradação desses materiais, logo se pautou na determinação da demanda da terra de absorção de carbono. Assim, como sumidouros, adotaram-se as florestas e o oceano [11]. Utilizou-se uma adaptação do modelo proposto por Marrero et al. [12] (Eq. 1):

$$
P E_{R S U}=G_{R S U} \times E_{R S U} \times \frac{F}{100} \times \frac{e f}{A}
$$

Onde:

$\mathrm{PE}_{\mathrm{RSU}}=$ Pegada Ecológica dos Resíduos Sólidos Urbanos (gha/ano);

$\mathrm{G}_{\mathrm{RSU}}=$ Geração de Resíduos Sólidos Urbanos (t/ano);

$\mathrm{E}_{\mathrm{RSU}}=$ Fator de emissão de $\mathrm{RSU}\left(0,244 \mathrm{t} \mathrm{CO}_{2} / \mathrm{t}\right)$ [13];

$\mathrm{F}=$ Porcentagem de absorção de $\mathrm{CO}_{2}$ pelas florestas (72\%) [11]; ef $=$ Taxa de sequestro de $\mathrm{CO}_{2}$ da biomassa vegetal $\left(0,4 \mathrm{~kg} \mathrm{CO}_{2}\right.$ $\mathrm{m}^{-2}$ ano $^{-1}[14]$;

$\mathrm{A}=$ Fator de equivalência das terras de absorção de carbono (1,26 gha/wha) [11].

Para a determinação da biocapacidade local foram consideradas as áreas disponíveis para assimilação do dióxido de carbono emitido durante a degradação dos RSU, para tanto, tomou-se como referência as áreas florestais, visto esses ecossistemas apresentarem o mais significativo potencial de sequestro e armazenamento de carbono [15]. Assim, tomaram-se como referência as áreas de Caatinga preservada no município em estudo, usando como base os resultados do monitoramento por satélite da Caatinga [16]. Para a determinação da biocapacidade, utilizou-se a equação proposta por Liu et al. [17] (Eq. 2):

$$
B=\sum A x F \text { e } x \text { Fp }(g h a)
$$

Onde:

$\mathrm{B}=$ Biocapacidade (gha)

$\mathrm{A}=$ Área local bioprodutiva existente (ha)

$\mathrm{Fe}=$ Fator de equivalência das terras de absorção de carbono $(1,26$ gha/wha) [11];

$\mathrm{Fp}=$ Fator de rendimento [14].

O Saldo Ecológico foi determinado através da equação proposta por Liu et al. [17] (Eq. 3):

$$
S D=B C-P E
$$

Onde:

$\mathrm{SD}=$ Saldo Ecológico;

$\mathrm{BC}=$ Biocapacidade da área;

$\mathrm{PE}=$ Pegada Ecológica.

\section{Resultados e discussões}

Observa-se uma taxa geração de RSU per capita em Poço das Trincheiras da ordem de 0,57 quilogramas por dia (Tabela 1). Valor superior aos estimados por Colvero et al. [18] para municípios do mesmo porte no estado de Goiás, com população total entre 10.001 a 20.000 habitantes, no qual essa taxa ficou em $0,54 \mathrm{~kg} / \mathrm{hab} /$ dia. A taxa de geração de RSU per capita diária aqui 
encontrada é a mesma observada por Ramachandra et al. [19] para Deli, segunda maior cidade da Índia. O que atesta que embora possua uma pequena população urbana, Poço das Trincheiras detém uma considerável geração individual de RSU.

Tabela 1 - Variáveis de geração de RSU para o município de Poço das Trincheiras, Alagoas.

\begin{tabular}{cccc}
\hline Município & $\begin{array}{c}\text { Geração de RSU } \\
\text { (kg/hab/dia) }\end{array}$ & $\begin{array}{c}\text { Geração de } \\
\text { RSU (t/dia) }\end{array}$ & $\begin{array}{c}\text { Geração anual de RSU } \\
\text { por ano (t/ano) }\end{array}$ \\
\hline $\begin{array}{c}\text { Poço das } \\
\text { Trincheiras }\end{array}$ & 0,57 & 1,36 & 496,4
\end{tabular}

Fonte: Adaptado de do Plano Estadual de Resíduos Sólidos do Estado de Alagoas [10].

Em trabalho em seis cidades do Nepal, Das et al. [20] encontram taxas de geração per capita de RSU entre 0,27 e 0,46 $\mathrm{kg} / \mathrm{hab} / \mathrm{dia}$, valores bem inferiores aos observados para o município em estudo nesse trabalho. Embora seja um país em desenvolvimento, o Brasil, difere da maioria dos outros países com nível econômico similar no tocante a geração per capita de RSU, sendo comparado a alguns países desenvolvidos [21].

A geração de RSU por dia e anual mostraram valores relativamente baixos, influenciados pela baixa população urbana do município, corroborando com o pressuposto por Chen [22]. Outros municípios de Alagoas, com porte e população semelhantes a Poço das Trincheiras, apresentam para essas variáveis valores superiores, como é o caso de Senador Rui Palmeira (2,44 t/dia e 890,6 t/ano) [10].

No tocante a gravimetria dos resíduos, observou-se uma predominância da fração orgânica $(60,13 \%)$, seguida pelo papel e papelão $(13,74 \%)$. O vidro apresentou a menor percentagem dentro dos constituintes (1,34\%) (Figura 2). Como enfatizam Abdel-Shafy e Mansour [23], a composição do RSU apresenta variações significativas de um município para outro, assim como entre países. O que se deve aos diferentes estilos de vida, nível de desenvolvimento econômico, regulamentações de gerenciamento de resíduos e o grau de industrialização. Ainda segundo esses autores, o conhecimento da quantidade e a composição desses resíduos são fundamentais para a determinação do manejo a ser empregado.

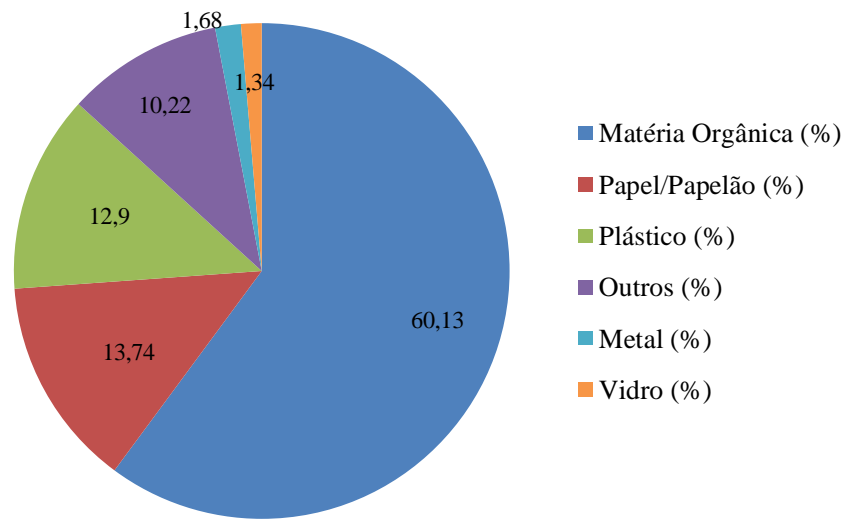

Figura 2 - Composição gravimétrica dos RSU do município de Poço das Trincheiras, Alagoas

Fonte: Adaptado de do Plano Estadual de Resíduos Sólidos do Estado de Alagoas [10].

Ao estudar a composição dos RSU da cidade indiana de Bangalore, Ramachandra et al. [19] também identificaram o predomínio de material orgânico, com uma percentagem de $72 \%$, sendo o papel e papelão o segundo item mais presente $(11,6 \%)$. A predominância de constituintes de origem orgânica em RSU é comum em economias em desenvolvimento ou de baixa renda, enquanto que em países desenvolvidos esses resíduos apresentam maiores frações de papel, plástico, metal e vidro [24]. Como já explicitado anteriormente, a gravimetria dos RSU é influenciada por diversos fatores, no Brasil merece atenção à influência do tamanho da população dos municípios e seu grau de desenvolvimento econômico; em cidades como São Paulo, a proporção dos constituintes dos RSU é semelhante às grandes cidades de países desenvolvidos, com proporção de material orgânico em torno de 53\% [3].

Diante da grande quantidade de matéria orgânica nos resíduos do município em estudo, o tratamento dessa fração via técnicas de processamento como compostagem (tratamento aeróbico) ou biometanização (tratamento anaeróbico), representam estratégias para a recuperação de energia e recursos contidos nesses resíduos [19]. Estratégias de reaproveitamento e reciclagem das outras frações também são pertinentes.

A partir dos resultados obtidos para a Pegada Ecológica verificou-se uma PE per capita de 0,1159 gha (Tabela 2), ou seja, cada habitante urbano demandou uma área de 0,1159 gha para absorver o $\mathrm{CO}_{2}$ da decomposição dos RSU gerados. Resultados superiores foram obtidos por Dai et al. [25] para a PE per capita dos RSU do bairro de Nanluoguxiang, Pequim, que foi de 0,192 gha. 
Tabela 2 - Pegada Ecológica (PE) per capita e total dos RSU de Poço das Trincheiras, Alagoas.

\begin{tabular}{ccc}
\hline Município & $\begin{array}{c}\text { Pegada Ecológica (PE) } \\
\text { per capita dos RSU } \\
\text { (gha) }\end{array}$ & $\begin{array}{c}\text { Pegada Ecológica (PE) } \\
\text { total dos RSU (gha) }\end{array}$ \\
\hline $\begin{array}{c}\text { Poço das } \\
\text { Trincheiras }\end{array}$ & 0,1159 & 276,84 \\
\hline
\end{tabular}

Song et al. [26] avaliando a PE do consumo de alimentos e geração de resíduos em 12.850 domicílios na China, obtiveram uma PE per capita de 0,4956 gha. Liu et al. [17] ao analisarem a PE da Universidade Politécnica de Tianjin observaram que os resíduos sólidos correspondiam a $16,56 \%$ da PE total da referida universidade.

Resultados que reforçam a necessidade da determinação da PE de RSU para melhor compreender o grau de impactos destes. Liu et al. [17] enfatizam que como por vezes a pegada ecológica não é facilmente compreendida, a PE per capita permite uma melhor interpretação dos resultados obtidos, facilitando assim a construção de um padrão.

A PE total dos RSU mostrou valores relativamente baixos (276,84 gha), o que se deve a pequena população urbana do município. Para um só bairro de Pequim, Dai et al. [25] estimaram uma PE de RSU da ordem de 5760 gha. Os valores aqui encontrados também foram inferiores aos reportados por Liu et al. [17] para a PE da Universidade Politécnica de Tianjin, que apresentou uma PE para os resíduos gerados de 771,30 gha.

Deve-se considerar que o presente estudo considerou apenas as emissões de $\mathrm{CO} 2$ na degradação de RSU. Pesquisadores como Ramachandra et al. [19] consideraram a liberação também de metano, visto a grande contribuição desse gás para o efeito estufa, logo, se consideradas as emissões de CH4, a PE dos RSU de Poço das Trincheiras poderia ser maior.

Através dos dados levantados, verificou-se que Poço das Trincheiras possui uma área de Caatinga preservada de 5200 hectares, o que equivale a uma biocapacidade de 26208 gha (Tabela 3). De forma sucinta, a biocapacidade reflete a capacidade dos organismos autotróficos de captar energia do sol por meio da fotossíntese e usar essa energia para gerar os recursos disponíveis para uso humano, bem como absorver as emissões de carbono geradas [27]. Assim, quanto maior a biocapacidade de uma área, maior será a sua capacidade de suporte. No presente estudo, a biocapacidade foi calculada pautando-se na área disponível para absorção de $\mathrm{CO}$, assim, para calcular a biocapacidade da geração de alimentos, usam-se outras áreas, como por exemplo, as disponíveis nas terras de lavoura e pastagens.

Tabela 3 - Pegada Ecológica (PE) per capita e total dos RSU de Poço das Trincheiras, Alagoas.

\begin{tabular}{ccc}
\hline Município & Biocapacidade (gha) & Saldo Ecológico (gha) \\
\hline $\begin{array}{c}\text { Poço das } \\
\text { Trincheiras }\end{array}$ & 26208 & 25931,16 \\
\hline
\end{tabular}

Observou-se um saldo ecológico de 25931,16 gha. Ao se comparar os resultados do saldo ecológico percebe-se um superávit ecológico, que é quando a pegada ecológica é inferior a biocapacidade, resultados que indicam que a área de terra bioprodutiva disponível pode suportar a demanda antrópica atual de geração de RSU [7-28]. Embora esse seja um resultado positivo, deve-se considerar que a biocapacidade calculada é responsável por absorver o $\mathrm{CO} 2$ emitido pelas demais atividades antrópicas no município, como por exemplo, as emissões geradas pela queima de combustíveis fósseis, logo, o saldo ecológico tende a ser bem menor. Ademais, ao passo que aumenta a urbanização, as emissões de gases de efeito estufa aumentam concomitantemente; todavia, as áreas de absorção desses gases diminuem, visto o avançado nível de degradação observado na Caatinga, já documentado para áreas próximas ao município de estudo, como reportado por Santos et al. [29] em municípios do alto sertão de Alagoas.

\section{Conclusões}

Poço das Trincheiras apresentou uma pequena produção de RSU anual, todavia, a produção per capita de seus habitantes se mostrou relativamente alta. Os resultados obtidos na gravimetria dos resíduos refletem o estilo de consumo comum nas cidades pouco desenvolvidas do Brasil, pautado na predominância da fração orgânica no montante geral de RSU.

A PE per capita e total dos RSU refletiram o mesmo comportamento observado para a produção desses resíduos. Esse indicador mostrou-se uma ferramenta importante para se compreender o grau de impacto da geração desses materiais, podendo sua utilização ser replicada para outras áreas.

A biocapacidade do município se mostrou capaz de

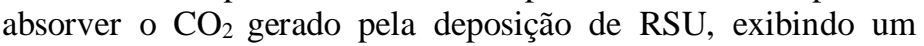
superávit ecológico.

\section{GENERATION OF URBAN SOLID WASTE IN THE BRAZILIAN SEMIARID: SUSTAINABILITY ANALYSIS FROM THE APPLICATION OF THE ECOLOGICAL FOOTPRINT}

ABSTRACT: The great urbanization and significant growth of the world population has led to a considerable increase in the generation of urban solid waste (USW). The destination of these materials is a matter of concern on the part of the international community, mainly because of its potential for environmental degradation. In this sense, considering the lack of studies on the generation of USW and the application of sustainability indicators in the generation of these materials in small Brazilian municipalities, this study aimed to evaluate the application of the 
Ecological Footprint (EF) to the USW generated in the city of Poço das Trincheiras, Alagoas. Data on generation and gravimetry were obtained through the State Solid Waste Plan of the State of Alagoas. The per capita and total EF were quantified, as well as the biocapacity and ecological balance of the study area. A per capita solid waste per capita generation rate of 0.57 kilograms per day was observed, totaling an annual production of 496.4 tons, with a predominance of organic material. The per capita $\mathrm{EF}$ of the USW was 0.1159 gha, and the total EF was 276.84 gha. The local biocapacity was estimated at 26208 gha, allowing an ecological surplus of 25931.16 gha. The Ecological Footprint has proved to be an important tool to understand the degree of impact of the USW generation.

Keywords: Environmental Quality. Biocapacity. Ecological Balance.

\section{Referências}

[1] WU, Y.; TAM, V. W.; SHUAI, C.; SHEN, L.; ZHANG, Y.; LIAO, S Decoupling China's economic growth from carbon emissions: Empirical studies from 30 Chinese provinces (2001-2015). Science of The Total Environment, Vol. 656 , p. $576-588,2019$

[2] KHANDELWAL, H.; DHAR, H.; THALLA, A. K.; KUMAR, S. Application of Life Cycle Assessment in Municipal Solid Waste Management: A Worldwide Critical Review. Journal of Cleaner Production, Vol. 299, p. 630-654, 2019.

[3] SANTOS, R. E.; SANTOS, I. F. S.; BARROS, R. M.; BERNAL, A. P.; TIAGO FILHO, G. L.; SILVA, F. D. G. B. Generating electrical energy through urban solid waste in Brazil: An economic and energy comparative analysis. Journal of Environmental Management, Vol. 231, p. 198-206, 2019.

[4] MAALOUF, A.; EL-FADEL, M. Towards improving emissions accounting methods in waste management: a proposed framework. Journal of Cleaner Production, Vol. 206, p. 197-210, 2019

[5] FRATTA, K. D. S. A.; TONELI, J. T. C. L.; ANTONIO, G. C. Diagnosis of the management of solid urban waste of the municipalities of ABC Paulista of Brasil through the application of sustainability indicators. Waste Management, Vol. 85, p. 11-17, 2019.

[6] XUN, F.; HU, Y. Evaluation of ecological sustainability based on a revised three-dimensional ecological footprint model in Shandong Province, China. Science of The Total Environment, Vol. 649, p. 582-591, 2019

[7] PENG, B.; LI, Y.; ELAHI, E.; WEI, G. Dynamic evolution of ecological carrying capacity based on the ecological footprint theory: A case study of Jiangsu province. Ecological Indicators, Vol. 99, p. 19-26, 2019.

[8] PAN, H.; ZHUANG, M.; GENG, Y.; WU, F.; DONG, H. Emergy-based ecological footprint analysis for a mega-city: The dynamic changes of Shanghai. Journal of Cleaner Production, Vol. 210, p. 552-562, 2019.

[9] IBGE - Instituto Brasileiro de Geografia e Estatística. @Cidades. Disponível em: https://cidades.ibge.gov.br/brasil/al/poco-das-trincheiras. Acesso em 09 de janeiro de 2019 .

[10] PIGIRS - Plano Intermunicipal de Gestão Integrada de Resíduos Sólidos. Região da Bacia Leiteira. Maceió: SEMARH, 2016. 58 p.

[11] MIKULČIĆ, H.; CABEZAS, H.; VUJANOVIĆ, M.; DUIĆ, N. Environmental assessment of different cement manufacturing processes based on Emergy and Ecological Footprint analysis. Journal of Cleaner Production, Vol 130, p. 213-221, 2016.
[12] MARRERO, M.; PUERTO, M.; RIVERO-CAMACHO, C.; FREIREGUERRERO, A.; SOLÍS-GUZMÁN, J. Assessing the economic impact and ecological footprint of construction and demolition waste during the urbanization of rural land. Resources, Conservation and Recycling, Vol. 117, p. 160-174, 2017.

[13] ALMASI, A. M.; MILIOS, L. Municipal waste management in Spain. European Environment Agency, 2013

[14] WACKERNAGEL, M.; MONFREDA, C.; MORAN, D.; WERMER, P.; GOLDFINGER, S.; DEUMLING, D.; MURRAY, M. National Footprint and Biocapacity Accounts 2005: The Underlying Calculation Method. Global Footprint Network, Oakland, USA, 2005. 33 p

[15] PAN, Y.; BIRDSEY, R.A.; FANG, J.; HOUGHTON, R.; KAUPPI, P.E., et al A large and persistent carbon sink in the world's forests. Science, Vol. 333, n. 6045 , p. $988-993,2011$

[16] INPE - Instituto Nacional de Pesquisas Espaciais. Monitoramento da Caatinga. Disponível em: http://geopro.crn.inpe.br/desmatamento.htm. Acesso em 09 de janeiro de 2019.

[17] LIU, H.; WANG, X.; YANG, J.; ZHOU, X.; LIU, Y. The ecological footprint evaluation of low carbon campuses based on life cycle assessment: A case study of Tianjin, China. Journal of Cleaner Production, Vol. 144, p. 266-278, 2017.

[18] COLVERO, D. A.; CARVALHO, E. H. D.; PFEIFFER, S. C.; GOMES, A. P Municipal solid waste generation assessment in the state of Goiás, Brazil: statistical data analysis. Engenharia Sanitaria e Ambiental, Vol. 22, n. 5, p. 931-941, 2017.

[19] RAMACHANDRA, T. V.; BHARATH, H. A.; KULKARNI, G.; HAN, S. S. Municipal solid waste: Generation, composition and GHG emissions in Bangalore, India. Renewable and Sustainable Energy Reviews, Vol. 82, p. 1122-1136, 2018.

[20] DAS, B.; BHAVE, P. V.; SAPKOTA, A.; BYANJU, R. M. Estimating emissions from open burning of municipal solid waste in municipalities of Nepal. Waste management, Vol. 79, p. 481-490, 2018.

[21] CETRULO, T. B.; MARQUES, R. C.; CETRULO, N. M.; PINTO, F. S MOREIRA, R. M.; MENDIZÁBAL-CORTÉS, A. D.; MALHEIROS, T. F. Effectiveness of solid waste policies in developing countries: A case study in Brazil. Journal of Cleaner Production, Vol. 205, p. 179-187, 2018

[22] CHEN, Y. Effects of urbanization on municipal solid waste composition Waste Management, Vol. 79, p. 828-836, 2018.

[23] ABDEL-SHAFY, H. I.; MANSOUR, M. S. M. Solid waste issue: Sources, composition, disposal, recycling, and valorization. Egyptian Journal of Petroleum, Vol. 27, n.4, p. 1275-1290, 2018

[24] AYODELE, T. R.; ALAO, M. A.; OGUNJUYIGBE, A. S. O. Recyclable resources from municipal solid waste: Assessment of its energy, economic and environmental benefits in Nigeria. Resources, Conservation and Recycling, Vol. 134, p. 165-173, 2018

[25] DAI, L.; XU, B.; WU, B. Assessing sustainable development of a historic district using an ecological footprint model: a case study of Nanluoguxiang in Beijing, China. Area, Vol. 49, n. 1, p. 94-105, 2017.

[26] SONG, G.; LI, M.; SEMAKULA, H. M.; ZHANG, S. Food consumption and waste and the embedded carbon, water and ecological footprints of households in China. Science of the Total Environment, Vol. 529, p. 191-197, 2015.

[27] MANCINI, M. S.; GALLI, A.; NICCOLUCCI, V.; LIN, D.; BASTIANONI, S.; WACKERNAGEL, M.; MARCHETTINI, N. Ecological Footprint: Refining the carbon Footprint calculation. Ecological Indicators, Vol. 61, n. 2, p. 390-403, 2016

[28] LU, Y.; LI, X.; NI, H.; CHEN, X.; XIA, C.; JIANG, D.; FAN, H. TemporalSpatial Evolution of the Urban Ecological Footprint Based on Net Primary Productivity: A Case Study of Xuzhou Central Area, China. Sustainability, Vol. 11, n. 1, p. 199, 2019. 


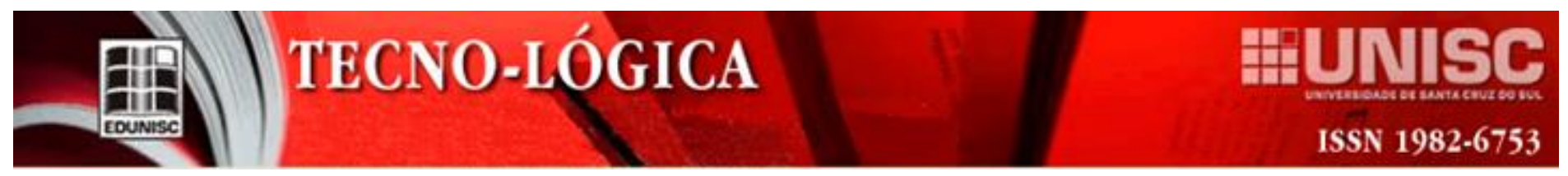

[29] SANTOS, J. P. O; SILVA JÚNIOR, J. M.; SILVA FILHO, J. A.; SILVA, J. L. C., RIBEIRO, J. K. R.; OLIVEIRA, F. I. F. Environmental Degradation and Risk of Desertification in Alto Sertão Region, Alagoas State, Brazil. Geama, Vol. 3, n. 4, p. 191-195, 2017. 